\title{
Some Considerations on Increase of Storage Capacity of Perpendicular Magnetic Hard Disk System
}

\author{
S. Yamamoto, A. Fujimura, I. Watanabe, and Y. Nakamura
}

\begin{abstract}
Contact perpendicular magnetic recording on both sides of a hard disk was attempted, using a head suspension mechanism employing a spring for vertical motion. A head-to-medium spacing of about $300 \AA$ was maintained at speeds up to $11 \mathrm{~m} / \mathrm{s}$ over all tracks on the disk. The results show that the distribution of magnetic properties of the recording layers on the hard disk are directly reflected in the envelope and the recording and playback characteristics. Data was successfully recorded at $100 \mathrm{kFRPI}$ and a track width of $60 \mathrm{\mu m}$.

I. Introduction

In the past the authors have conducted evaluations of the read/write characteristics of perpendicular magnetic recording systems by contact scanning of a single-pole head over flexible media prepared by sputter de-
\end{abstract}

S. Yamamoto, A. Fujimura, I. Watanabe, and Y. Nakamura are with Tohoku University.

From the Journal of the Magnetics Society of Japan (Nippon Oyo Jiki Gakkaishi), Vol. 15, No. 2, 1991, pages 179-182.

The copyright for the original Japanese article is held by the Magnetics Society of Japan. The copyright for the English version of this article as it appears here is held by the Institute of Electrical and Electronics Engineers, Inc.

TJMJ911024 position of a metal magnetic thin film onto polymer base film. However, flexible media in their present form suffer from microscopic surface roughness and curling at the time of medium fabrication. Conequently, it is not easy to obtain a playback signal with a satisfactory envelope, nor to maintain easily high pass-wear durability [1]. To study a way to achieve extremely small head-medium spacings using a flat medium with minimal surface roughness, we attempted contact scanning of a self-loaded single-pole head over hard disk media. This headmedium system yielded playback signals with a good envelope over the entire disk surface, as well as high pass-wear durability $[2,3]$. Such a recording system is thus far easier to use than one with flexible media [3] when experimenting with applications such as the recording and play-back of still image data. In this report, we describe studies of the possibilities for high-density recording using such a head-medium system.

II. Non-Flying Single-Pole Heads Capable of Accessing Both Sides of the Disk Medium

When using a head in contact with a hard disk, if a mechanism 


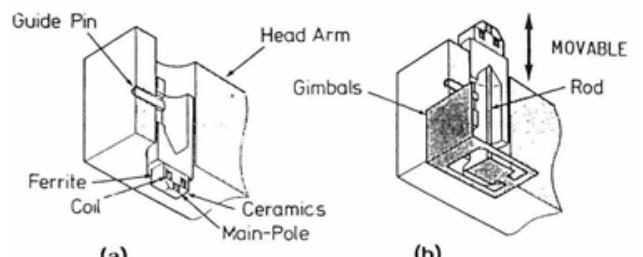

(a)

(b)

Fig. 1 Structure of the nonflying single-pole head. (a) Making contact under selfloading; and (b) loading by a spring.

is used that does not free the head to fly over the disk surface, then surface variations of the rotating disk must be $a b-$ sorbed by the head support mechanism. This is in contrast to conventional flying heads. The head support mechanism we are now using is completely different from ordinary assemblies based on head sliders. The single-pole head is mounted on the tip of an assembly which is free to slide vertically, as in Fig. 1(a). This is self-loaded (load under $1 \mathrm{~g}$ ) to make contact with the hard disk. The basic concepts behind the head support mechanism are as follows:

Head motion in lateral directions is restrained, but the head moves freely in the vertical direction to follow the medium surface; (2) The head tip is tapered to reduce the area of the head making contact with the disk, thereby preventing head flight; (3) By reducing the weight of the overall assembly including the head, the pressure exerted on the medium by the head is reduced, thereby preventing damage to both medium and head. However, when using contact by self-loading, only

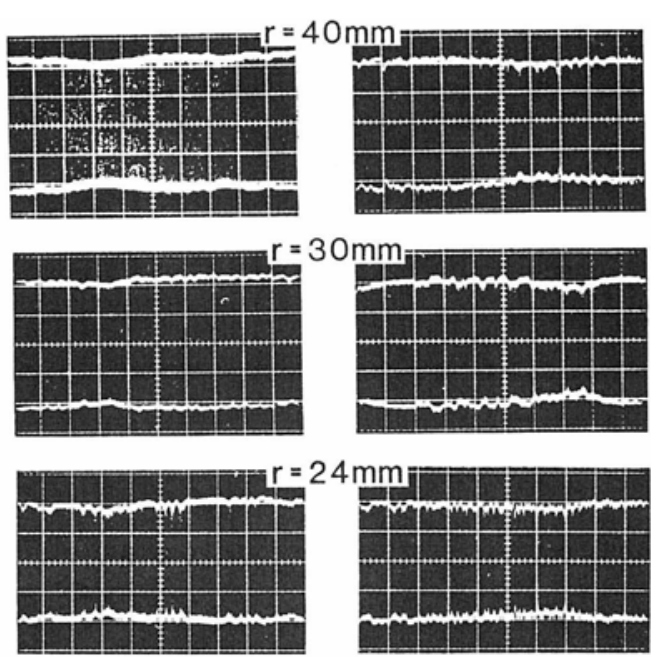

(a)

(b)

Fig. 2 Envelope of reproduced voltage at $50 \mathrm{kFRPI}$ and $2 \mathrm{~m} / \mathrm{s}$; $\mathrm{r}$ is the radius of the recorded track. (a) and (b) are for the head suspension mechanisms of Fig. 1 (a) and (b) respectively.

the surface of the medium below the head can be accessed. We therefore studied the use of gimbals at the back end of the head assembly to press the head against the medium. In this method, the spring force is adjusted to enable medium accessing from any direction. The prototype heads used had a mass of $50 \mathrm{mg}$, and the total mass of the movable head assembly was $700 \mathrm{mg}$; the spring force was $1800 \mathrm{mg}$, and accessing of the bottom surface of the medium was studied. The hard disk was based on flat glass substrate, and had an average surface roughness $\left(R_{a}\right)$ of about $25 \AA$.

Fig. 2 shows envelopes of the reproduce voltage over a complete revolution of a 3.5 " disk, at the innermost, outermost and at intermediate tracks, 
recording and reading at $2 \mathrm{~m} / \mathrm{s}$ and 50 kFRPI. Fig. 2 (b) denotes accessing of the bottom side of the medium using spring force, Fig. 2 (a) indicates accessing of the upper medium surface using $0 \mathrm{mg}$ spring force. Even when using the spring, a good state of head-medium contact is maintained over the entire disk surface, and a playback voltage without envelope fluctuation is obtained. Glass dummy heads with the same tapered tip as the actual prototype heads were also scanned in contact over the hard disk surface using the same support mechanism, and optical interferometry was used to investigate the state of headmedium contact. The region in which the head tip made contact with the medium was an oval area approximately $200 \mu \mathrm{m}$ long (track width direction) and $100 \mu \mathrm{m}$ wide (direction of medium motion). The head-medium spacing in this oval-shaped region was approximately $300 \AA$.

Eig. 3 presents the results of studies of the relation between the state of head contact and the head-medium relative velocity. After first recording signals at $5 \mathrm{kERPI}$ and $2 \mathrm{~m} / \mathrm{s}$, the velocity was raised to 11 $\mathrm{m} / \mathrm{s}$ and the playback voltage was measured. The vertical axis indicates the reproduce voltage (the average value over a revolution) divided by the relative velocity. Both when the spring was used to access the lower surface of the medium (filled circles), and when the upper surface was accessed at a spring force of $0 \mathrm{mg}$ (empty circles), there was no change in the reproduce voltage per unit velocity for velocities of $11 \mathrm{~m} / \mathrm{s}$ or less. This in turn indicates that the head-medium spacing

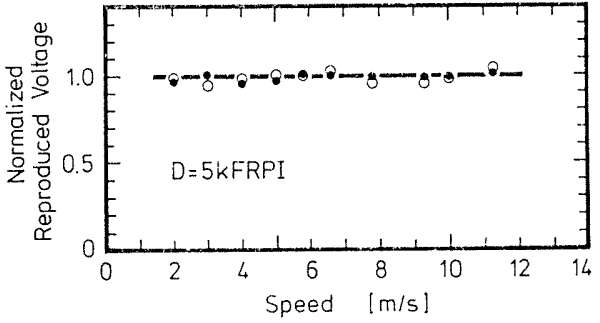

Fig. 3 Head-disk relative velocity dependence of the reproduce voltage, normalized by the velocity. Empty and filled circles denote the head suspension mechanisms of Fig. $1(a)$ and (b) respectively.

does not increase as the velocity is increased. Moreover, as there is almost no difference in the recording density characteristics measured using the two support methods, the head-medium spacing is nearly the same for both. Virtually no change was observed in the signal envelope with varying relative velocity for velocities of $11 \mathrm{~m} / \mathrm{s}$ or less, thereby confirming that the head can follow the disk surface and maintain a good state of contact.

To summarize, a springbased head support method such as that illustrated in Fig. 1(b) may be used in contact recording on both surfaces of a hard disk, at a spacing of approximately $300 \AA$.

III. Read/Write Characteristics A. Effect of medium magnetic
properties on signal envelope By introducing a method for contact recording on hard disks, it was possible to obtain a modulation-free envelope. However, fluctuations in the envel- 

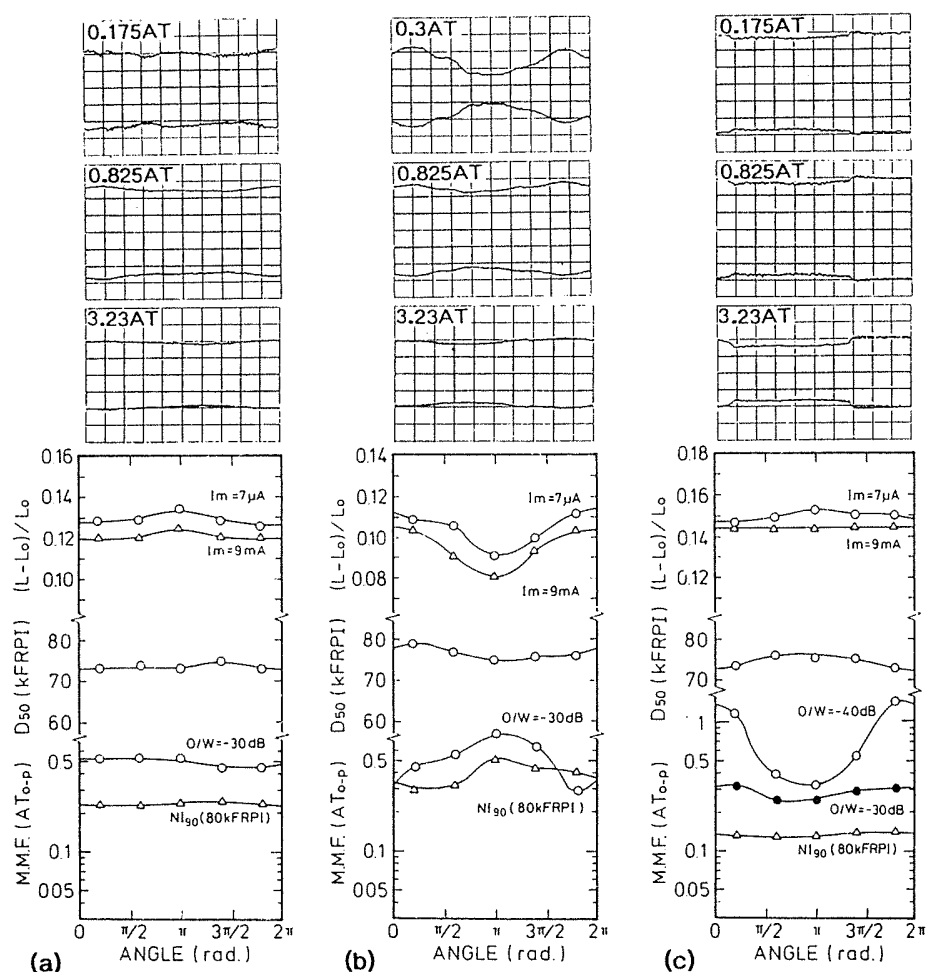

Fig. 4 Figures at the top are reproduce voltage envelopes for one disk revolution. At the bottom are change in head inductance, $\mathrm{D}_{50}$, overwrite $\mathrm{MMF}$, and $\mathrm{NI}_{90}$ as functions of angular position corresponding to the envelope diagrams above.

ope over a given track are observed for some types of media. Envelope fluctuations may be broadly divided into three types, as in Fig. 4. The diagrams in the figure show several different measured results over a single rotation of the medium. The relative change in inductance $\left(L-L_{0} / L_{0}\right)$ of a single-pole head in the presence and absence of the medium is obtained from $\mathrm{L}_{0}$, the inductance of the head by itself, and $L$, the inductance when the head is brought into contact with the medium as when recording and reading signals. The higher the permeability of the soft magnetic backing layer of the medium, the larger will be the relative change in inductance [4]. The recording density $D_{50}$ is where the reproduce voltage falls to one-half its low-density value. The recording Magneto-Motive-Eorce (MMF) at which the playback voltage is $90 \%$ of the saturation value is $\mathrm{NI}_{90}$. The MMF at which 30 kFRPI signals overwritten by 70 kFRPI signals are attenuated by $-30 \mathrm{~dB}$ compared with the playback voltage prior to overwriting was also measured. In the case of medium (a), there are no fluctuations with the position on the medium in either the relative change in induct- 
ance or in the other recording and playback characteristics, and an envelope free of fluctuations is obtained. In contrast, in the regions of medium (b) where the playback voltage envelope is decreased, the relative change in inductance is small, and the overwrite and recording sensitivity are also reduced. When the recording MMF is high, a relatively flat envelope is obtained, but at low recording MME's there are large fluctuations in the envelope. Because of this, it is expected that in regions where the envelope playback voltage is low, the permeability of the soft magnetic backing layer of the medium is reduced, so that satuation recording is harder to achieve. In medium (c), on the other hand, the relative change in inductance is nearly uniform over an entire rotation of the medium, and we may conclude that there is almost no fluctuation with position in the magnetic properties of the soft magnetic backing layer. In regions where the playback voltage envelope is low, $\mathrm{D}_{50}$ is increased and signals are more easily overwritten, allowing us to infer that in such regions the $\mathrm{Co}-\mathrm{Cr}$ layer coercivity in the perpendicular direction is low compared to other areas. One possible reason for the variation in magnetic properties with location on the disk is the creation of a temperature distri-bution in the glass substrate during sputter deposition of the film medium. When aluminum leaf was inserted between the glass substrate and the substrate holder to improve thermal conduction, fluctuations in the envelope were greatly reduced. In this way, a satisfactory state of head-medium contact can be stably maintained when using hard disk media, and the distribution of magnetic properties in the medium is very directly reflected in the read/write characteristics. Hence caution must be exercised during medium fabrication to ensure that no fluctuations with position appear in the magnetic properties of the medium magnetic layer.

\section{B. Possibilities for high- density recording}

The relation between linear and track densities and the occurrence of errors in contact recording onto a hard disk was also investigated. The singlepole head used had a main pole thickness of $0.19 \mu \mathrm{m}$, a track width of $100 \mu \mathrm{m}$ and an $\mathrm{Fe}-$ $\mathrm{Si} / \mathrm{SiO}_{2}$ multilayer film as the main pole. The medium was a hard disk with a Co-Cr layer having perpendicular coercivity of 1270 oe, thickness $0.1 \mu \mathrm{m}$, saturation magnetization $385 \mathrm{emu} / \mathrm{cc}$ and perpendicular magnetic anisotropy field of $4 \mathrm{kOe}$ The $\mathrm{Cu}-\mathrm{Mo}$ permalloy soft magnetic backing layer was $0.78 \mu \mathrm{m}$ thick. The medium surface was covered with a protective $\mathrm{SiO}_{2}$ layer $80 \AA$ thick.

Fig. 5 plots the $\mathrm{C} / \mathrm{N}$ ratio of $2 \mathrm{~F}$ signals and the peak shift for a repeated MFM "110" pattern at various linear recording densities, with the data transfer rate held constant at 10 Mbits/s. The $D_{50}$ and the recording density at which the peak shift was $20 \%$ were both 100 $\mathrm{kFRPI}$. Cases in which $29 \mathrm{k}$ Bytes of data were recorded and reproduced without error are denoted by empty circles; cases in which errors occurred are indicated by filled circles. At linear densi- 


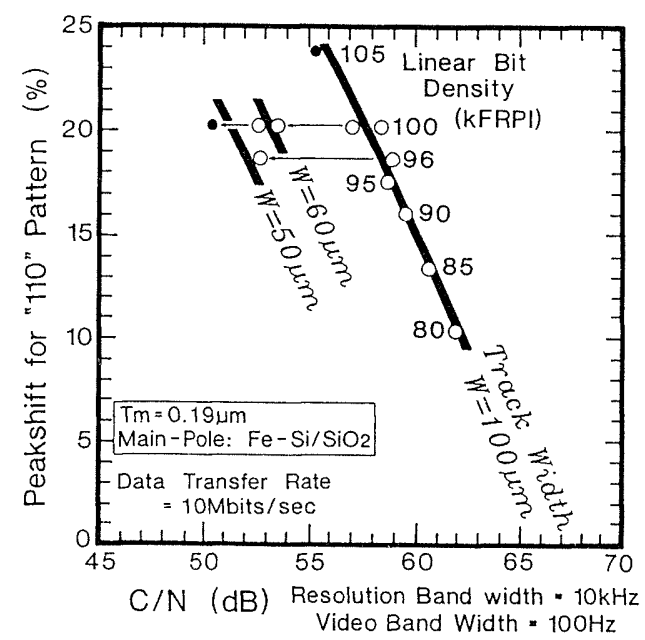

Eig. 5 Peak shift, $C / N$ and error rate measured at various recording densities.

ties below $100 \mathrm{kFRPI}, 29 \mathrm{k}$ Bytes of data could be written and reproduced without errors occurring. In another test, data was first written to the medium, and then the head was moved in the track width direction and the edge of the recorded track was erased to reduce its width.

After words, the head was again centered on the track and the data reproduced. The resulting $\mathrm{C} / \mathrm{N}$ at different linear densities changed as indicated by the arrows in the diagram. In these experiments, the noise level was limited mainly by system noise, so that the $\mathrm{C} / \mathrm{N}$ varied nearly in proportion to the track width; no errors occurred even when the track width was reduced to 60 $\mu \mathrm{m}$. When the track width was further reduced to $50 \mu \mathrm{m}, 10$ error bits appeared at a density of $100 \mathrm{kFRPI}$, but none occurred when recording at $96 \mathrm{kFRPI}$. Based on the above experimental results, recording and playback with MFM modulation of still image data, at a data transfer rate of $10 \mathrm{Mbits} / \mathrm{s}$ and with the amount of data increased to $768 \mathrm{k}$ Bytes, was attempted. At $100 \mathrm{kFRPI}$ and a track width of $60 \mu \mathrm{m}$, error-free recording and playback was possible, and recording at a higher density than when using flexible disk media was achieved [5].

In these experiments, the $\mathrm{C} / \mathrm{N}$ of $2 \mathrm{~F}$ signals for a linear density of $100 \mathrm{kFRPI}$ and a track width of $50 \mu \mathrm{m}$ was $50.5 \mathrm{~dB}$; assuming a flat noise-frequency characteristic (the cutoff frequency of the read system was 10 $\mathrm{MHz}$ ), the $\mathrm{S} / \mathrm{N}$ is estimated to be $20.5 \mathrm{~dB}$ (rms/rms). With this $\mathrm{S} / \mathrm{N}$ and a peak shift of $20 \%$, theoretically there should be no errors in the recording and playback of $29 \mathrm{k}$ Byte or so of data[6], but in these experiments errors did occur. Dropout and medium defects are presumed to be the causes of these errors.

IV. Conclusion

A spring-based head support mechanism was introduced to enable contact recording onto both surfaces of a hard disk using a single-pole head. In this method, highly stable headmedium contact can be maintained, and spatial distributions of the magnetic properties of the medium tended to be reflected directly in the read/ write characteristics. On performing high-density recording experiments using hard disk media with minimal variation in magnetic properties, error-free recording and playback of $768 \mathrm{k}$ Bytes of data was possible at a linear recording density of 100 kFRPI and track width of $60 \mu \mathrm{m}$, 
and high-density recording was achieved more readily than when using flexible recording media. Hereafter, we hope to achieve higher areal densities through development of singlepole heads with higher sensitivity and by reducing dropouts in the hard disk media.

\section{References}

[1] M. Isurugi, Y. Nakamura and S. Iwasaki, IEEE Trans. Magn., MAG-24, 2658, 1988 .

[2] Y. Nakamura, K. Ouchi and I. Watanabe, J. Magn. Soc. Jpn., $14,135,1990$.

[3] S. Yamamoto, I. Watanabe, K. Ouchi and Y. Nakamura, IEICE Tech. Rep., MR90-14, 31, 1990. [4] I. Watanabe, K. Ouchi and $Y$. Nakamura, Dig. 10th Annual Conf. Magn. Jpn., 6pA-12, 1986.

[5] S. Yamamoto, H. Muraoka, Y. Nakamura and S. Iwasaki, J. Magn. Soc. Jpn., 13, 273, 1989. [6] Y. Tahara, Y. Miura and Y. Ikeda, Trans. IECEJ, J59-C, 607, 1976. 